\title{
Un exemple de collaboration entre les sociétés suisses de disciplines médicales
}

Groupe de travail

multidisciplinaire*

\begin{abstract}
Introduction
Devant le développement rapide et hétérogène des unités de soins intermédiaires (U-IMC) à travers la Suisse, les différentes sociétés de disciplines médicales impliquées dans leur gestion ont identifié le besoin d'élaborer des directives nationales communes définissant un cadre formalisé de fonctionnement de ces unités. Sous l'impulsion de la Société Suisse de Médecine Intensive, la Société Suisse d'Anesthésiologie et de Réanimation, la Société Suisse de Cardiologie, la Société Suisse de Chirurgie, la Société Suisse de Chirurgie pédiatrique,the Swiss Federation of Clinical Neuro-Societies, la Société Suisse de Médecine Interne Générale, the Swiss Society of Neonatology et la Société Suisse de Pédiatrie ont mandaté un groupe de travail multidisciplinaire pour élaborer ces
\end{abstract}

\section{Champs couverts par les directives}

Ces directives définissent les objectifs et la mission des U-IMC et discute le type de malades susceptible d'être admis dans de telles unités. Elles établissent des exigences minimales en termes de locaux, d'organisation, de personnel, d'équipement et de moyens en matériel. Les recommandations sont le résultat d'un consensus dont le but est d'assurer un standard minimal tout en permettant l'application des directives.

\section{Organisation générale et indicateurs chiffrés}

En plus de recommandations sur le fonctionnement général des unités, les directives préconisent la collecte d'indicateurs chiffrés tant structurels (nombre de lits, taille des locaux, etc.) qu'opérationnels (res-

\section{Il y a le besoin de définir des standards pour le fonctionnement de ces unités dans des conditions de sécurité.}

Correspondance: Dr Yvan Gasche c/o Geschäftsstelle SGI, IMK Münsterberg 1 CH-4001 Bâle sources humaines, durée de séjour, etc.) pour permettre d'assurer un contrôle de qualité minimal et un «benchmarking» adéquat. clefs (les directives complètes sont disponible en ligne). Ces directives définissent les besoins minimaux des U-IMC. Approuvées par les organes officiels de toutes les sociétés impliquées dans leur élaboration, elles entreront en vigueur début 2014.

\section{Taille des unités, nombre et qualité des patients} Les directives définissent la taille minimale des uni-

\footnotetext{
* Groupe de travail multidisciplinaire (par ordre alphabétique): Gérald Berthet, Société Suisse de Pédiatrie

Thomas Brack, Société Suisse de Médecine Interne Générale

Léo Bühler, Société Suisse de Chirurgie

Tiziano Cassina, Société Suisse d'Anesthésiologie et Réanimation

Jolanda Contartese, Société Suisse de Médecine Intensive

Philippe Eckert, Société Suisse de Médecine Intensive

Yvan Gasche, Société Suisse de Médecine Intensive

Pierre-Frédéric Keller, Société Suisse de Cardiologie

Angelika Lehmann, Société Suisse de Médecine Intensive

Martine Louis Simonet, Société Suisse de Médecine Interne Générale

Marie-Luise Mono, Swiss Federation of Clinical Neuro-Societies

Marion Mönckhoff, Société Suisse de Néonatologie

Sonia Saudan, Société Suisse d’Anesthésiologie et Réanimation

Stefan Schmid, Société Suisse de Chirurgie

David Tüller, Société Suisse de Cardiologie

Werner Z'Graggen, Swiss Federation of Clinical Neuro-Societies
} tés (nombre de lits), le nombre de journées de soins minimal indispensable ainsi que la gravité des malades susceptibles d'être admis dans une telle structure. Une catégorisation des malades permettant de définir la charge en travail et leur gravité est préconisée afin de pouvoir optimaliser l'utilisation des ressources.

\section{Architecture, locaux et équipements}

Les directives définissent des exigences minimales en termes d'architecture et de locaux ainsi que d'équipements afin de permettre au personnel d'assurer dans de bonnes conditions les soins et de garantir la sécurité des malades.

\section{Ressources humaines}

Les directives définissent des exigences quantitatives et qualitatives en ce qui concerne le personnel médical et infirmier. Elles définissent la qualification mini- 


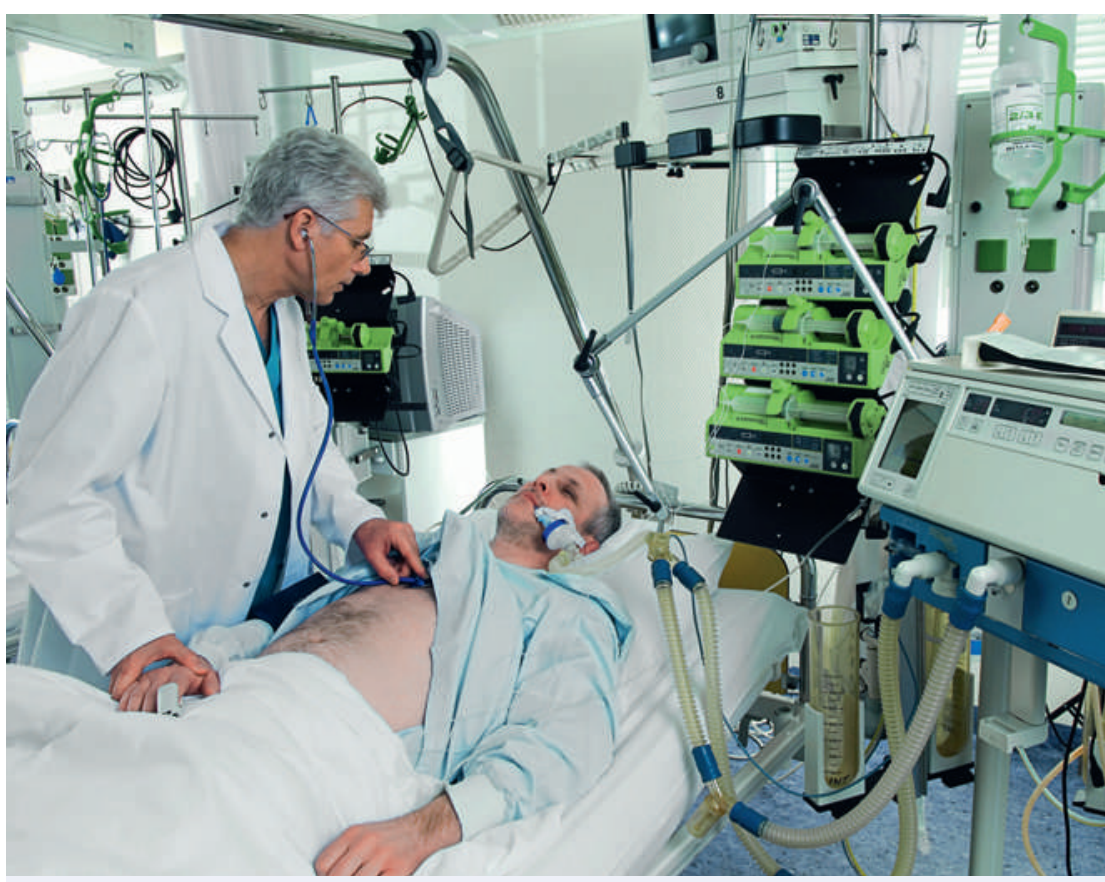

Des unités de soins intermédiaires ont été développés pour les patients, dont la gravité ne justifie pas qu'ils soient admis dans des unités de soins intensifs.

male des responsables médicaux et infirmiers. Elles précisent les requis minimaux concernant la hiérarchie médicale, l'organisation des gardes et des piquets médicaux. La dotation minimale en personnel infirmier de terrain et sa qualification sont également définies dans les directives.

\section{Processus d'application des directives}

Les directives représentent un standard contraignant pour la reconnaissance des U-IMC par les sociétés cosignataires. Le processus de reconnaissance sera mené par la commission de reconnaissance des U-IMC (CRUIMC). Cette dernière sera constituée de membres médecins et infirmiers choisis par les différentes sociétés. L'activité de la commission sera définie au sein d'un règlement de fonctionnement approuvé par les sociétés de disciplines.

Pour une période transitoire de cinq ans, les unités déjà existantes au moment de l'entrée en vigueur des directives pourront déposer un dossier auprès de la commission afin d'obtenir une reconnaissance provisoire sur examen du document. La reconnaissance provisoire deviendra définitive après que la procédure complète de reconnaissance aura été menée.

\section{Conclusion}

Ces directives pour la reconnaissance des unités de soins intermédiaires suisses sont le résultat d'un effort multidisciplinaire motivé par le besoin de définir des standards pour le fonctionnement de ces unités dans des conditions de sécurité. Les sociétés de disciplines cosignataires ont spécifiquement défini dans ces directives les conditions cadres pour garantir la prise en charge optimale des patients de soins intermédiaires selon les objectifs de soins médicoinfirmiers spécifiques. Les recommandations émises permettront aux hôpitaux de définir les ressources, les structures et l'organisation de leurs unités et d'assurer le contrôle de qualité de leurs structures de soins intermédiaires. Ceci permettra également de définir de manière standardisée une activité médicale grandissante au sein des hôpitaux qui n'est actuellement pas caractérisée en terme de remboursement.

Trouvez les directives online: www.sgi-ssmi.ch 